\title{
Highly efficient cardiac differentiation and maintenance by thrombin-coagulated fibrin hydrogels enriched with decellularized porcine heart extracellular matrix
}

\author{
Fatemeh Navaee $^{1}$, Philippe Renaud ${ }^{1}$, Thomas Braschler ${ }^{2 *}$ \\ 1. Laboratory of Microsystems 4, STI-IMT, Station 17, EPFL, 1015 Lausanne, Switzerland \\ 2. Department of Pathology and Immunology, Faculty of Medicine, University of Geneva, CMU, Rue \\ Michel-Servet 1, 1211 Genève 4, Switzerland \\ * Corresponding author: Thomas.braschler@unige.ch, Tel: +41223794170
}

\begin{abstract}
We provide a blend of cardiac decellularized extracellular matrix (dECM) from porcine ventricular tissue and fibrinogen for the formation of an in-vitro 3D cardiac cell culture model. Rapid and specific coagulation with thrombin allows gentle inclusion of cells while avoiding sedimentation during formation of the dECM-fibrin composite. We use the system in co-culture with Nor-10 fibroblasts to enhance cardiogenic differentiation of the H9c2 myoblast cell line. The combination of co-culture and appropriate substrate allows to abrogate the use of retinoids, classically considered necessary for cardiogenic H9c2 differentiation. Further enhancement of differentiation efficiency is obtained by 3D embedding. We then proceed with culture of rat neonatal cardiomyocytes in the 3D system. While for H9c2 cells, the collagen content of the dECM was the key factor required for efficient differentiation, the use of dECM-fibrin has specific advantages regarding the culture of neonatal cardiomyocytes. Calcium imaging and analysis of beating motion both indicate that the dECM-fibrin composite significantly enhances recovery, frequency, synchrony and maintenance of spontaneous beating, as compared to various controls including matrigel, pure fibrin and collagen I, but also a fibrin-collagen I blend.
\end{abstract}

Keywords: dECM-fibrin hydrogel; neonatal cardiomyocyte culture; H9c2 cell differentiation; beating synchrony; 3D co-culture 


\section{Introduction}

Cardiovascular disease, and in particular myocardial infarction, is the first cause of mortality by representing $31 \%$ of global death[1]. In myocardial infarction, cardiac ischemia leads to oxygen deficiency, cell injury and ultimately cell death[2]. After myocardial infarction, structural and functional remodeling in the remaining heart tissues occurs[3]. As adult cardiomyocytes have limited regeneration capability, finding strategies to enhance heart tissue regeneration can help patients recover after myocardial infarction[4]. Our aim here is to provide a simple, yet highly efficient invitro model for cardiomyocyte differentiation and maintenance. We design this model to simultaneously recapitulate three main dimensions of the native cardiomyocyte environment[5][6]: extracellular matrix cues[7], mechanical stiffness[8] and interaction with stromal fibroblasts[9]. This model has potential applications in drug screening, simplifies generation of cardiomyocytes and may provide building blocks for cell transplantation in regenerative medicine.

Our first cardiomyocyte environment element is the choice of a specific cardiac extracellular matrix. We opt here for the use of porcine decellularized extracellular matrix (dECM) of cardiac origin to provide organ-specific cues[10], with reinforcement by thrombin-coagulated fibrin[11] for improvement of mechanical properties[12].

Myocardial dECM has indeed been shown to powerfully support cardiomyocyte differentiation and maturation in many cell lineages, including human embryonic stem cells and rat neonatal ventricular cardiomyocytes[7]. Moreover, porcine cardiac dECM hydrogels are ultrastucturally similar to their human analogs[13] and known to provide functional benefits after myocardial infarction in animal models[14]. Preservation of part of the organspecific cues[15][16], [17], including a fraction of the growth factors contained in the dECM[7][18], is thought to be responsible for correct organ-specific cellular differentiation.

Besides dECM, a variety of synthetic and natural matrices have been used for cardiomyocyte culture[19][20][21][22][23]. Collagen I, and mixtures of collagen I with matrigel have in particular been reported to be supportive of spontaneous synchronized in-vitro contractions, albeit requiring mixed cardiac cultures with both contractile and non-contractile cells, either primary or derived from induced pluripotent stem cells[20][24][23]. Invivo, collagen foams and matrigel were further shown to enhance engraftment efficiency of the cardiac model cell line $\mathrm{H} 9 \mathrm{c} 2[25]$. With regard to the extracellular matrix element, an important aim of this study is therefore to 
quantify possible advantages of the proposed dECM-fibrin composite over readily available commercial controls such as collagen I or matrigel.

The second element of our in-vitro cardiomyocyte niche model is the achievement of appropriate mechanical stiffness. Pure cardiac dECM hydrogels have long gelation times[18] and mechanical properties far below native cardiac tissue[26][7]. This is a practical problem, since sedimentation during the extended gelling time will make cell seeding difficult[27]. More importantly, however, it is known that the differentiated phenotype of heart cells is the most prominent on substrates with stiffness comparable to that of the native heart[8].

Various approaches to reinforce cardiac dECM are available, and include for instance covalent crosslinking[28] or combination with additional hydrogels[29]. Williams, et al. combined the two approaches by blending fibrin with dECM from neonatal and adult rat hearts, followed by crosslinking with transglutaminase[30]. However, the lack of specificity of transglutaminases rises concerns about cellular toxicity in-vitro[30] and possible side effects such as autoimmune reaction due to altered self-epitopes in-vivo[31]. To avoid such side reactions, we here use fibrin obtained by coagulation of fibrinogen with human thrombin[11] to raise the level of stiffness in dECM-fibrin blends in a more specific and safer way. The use of fibrin as opposed to synthetic polymers[29] is motivated by the reported increased efficiency of cardiac reprogramming in the presence of this biopolymer[32].

Finally, the third element of the cardiomyocyte niche to be addressed in this study is the stromal support by fibroblasts. The heart indeed consists only to some $30 \%$ of contractile cardiomyocytes[9], the remainder being mainly endothelial cells and fibroblasts[33]. The fibroblast cells in native heart tissue have a major and complex roles in cardiac development, myocardial structure, cell signaling, and electromechanical function[9][34]. In-vitro, co-culture with fibroblasts has been shown to enhance skeletal muscle regeneration from myogenic progenitor cells[35] as well as electrophysiological maturation of iPSC-derived cardiomyocytes [36]. We hypothesize here that co-culture with fibroblasts in the context of a mechanically and biologically relevant matrix could be used to enhance differentiation of cardiomyocytes from myoblast progenitor cells.

We use the H9c2 cell line as a model system for cell differentiation[37]. Originally derived from rat embryonic ventricular heart tissue, this spontaneously immortalized line differentiates towards skeletal muscle upon reaching confluence[37]. Yet, upon exposure to retinoic acid, cardiac differentiation can be recovered[38]. The cardiac differentiation efficiency of $\mathrm{H} 9 \mathrm{c} 2$ cells can be further enhanced by the presence of fibroblast-derived matrix in 
addition to the retinoic acid[39]. Our goal is to optimize cardiogenic differentiation of H9c2 by combining fibroblast support with appropriate mechanical and matrix cues in composites of coagulated fibrin and porcine cardiac dECM hydrogels. We aim at replacing the exogenous retinoic acid by endogenous cues to simplify the differentiation protocol. This allows to avoid the presence of the strong, widespread effects of retinoids on gene expression in screening and gene expression profiling experiments[40].

Regarding the development of a cardiac in-vitro niche with relevant cellular, mechanical and matrix components, a major aim is also to better support physiological electromechanical activity and synchronization of primary neonatal cardiomyocytes. Hence, we investigate synchrony, beating rate and recovery time of neonatal cardiomyocytes in various hydrogel compositions including fibrin, collagen I and matrigel, but also the composites fibrin-collagen I and dECM-fibrin. This allows us to further refine our comprehension of niche effects, with relevance to culture of primary neonatal cardiomyocytes and the optimal definition of tissue engineering and transplantation matrices.

2. Materials and methods

2.1. Extracellular matrix decellularization and characterization

Decellularized porcine cardiac extracellular matrix forms the basis of the dECM-fibrin hydrogel studied in this report. The procedure for decellularization of the cardiac tissue was based on published literature[10]. Briefly, porcine heart tissue was obtained from a local slaughterhouse and the ventricles cut into pieces of about $1 \mathrm{~mm}$ in thickness. The pieces were rinsed with deionized water and then stirred in 1\% Sodium Dodecyl Sulfate (SDS) in a phosphate buffered saline (PBS) solution for $48-72 \mathrm{~h}$ at $4^{\circ} \mathrm{C}$, followed by $1 \%$ Triton $\mathrm{X}-100$ for an additional $30 \mathrm{~min}$. Finally, the preparation was stirred in deionized water overnight and freeze-dried. The dECM powder thus obtained was suspended in $0.1 \mathrm{M} \mathrm{HCl}$, followed by pepsin (Sigma P6887) digestion for 72 hours (100mg of dECM and 10mg of pepsin per $1 \mathrm{~mL}$ of $\mathrm{HCl}$ )[10]. The $\mathrm{pH}$ was then adjusted to 7.4 by gradual addition of $\mathrm{NaOH}$, yielding a solution of $100 \mathrm{mg} / \mathrm{mL}$ dECM. dECM stock solution was finally obtained by adding Dulbecco's Modified Eagle Medium DMEM (Thermofisher, cat\# 41965) to reach a final dECM concentration of $50 \mathrm{mg} / \mathrm{mL}$.

To verify the extent of decellularization, the residual DNA content in both native and decellularized tissue was measured. For this, dECM (respectively intact cardiac tissue) was dissolved in lysis buffer (0.5 M EDTA pH 8.0, $0.5 \%$ SDS) and $100 \mu \mathrm{g} / \mathrm{mL}$ proteinase K (Sigma, P4850) overnight at 55 [41]. The resulting suspension was vortexed, and proteins precipitated with phenol-chloroform, followed by centrifugation at $13700 \mathrm{rpm}$ for $40 \mathrm{~min}$. 
DNA was then isolated by recovery of the top (aqueous) phase, followed by addition of $0.5 \mathrm{~mL}$ ethanol, resuspension in deionized water, and quantification by Carry 50 spectrophotometer using a quartz cuvette at $260 \mathrm{~nm}$. For further quantification of dECM composition, collagen and Glycosaminoglycan (GAG) content in the dECM were measured using the Sircol ${ }^{\mathrm{TM}}$ Soluble Collagen Assay kit and Blyscan Sulfated Glycosaminoglycan Assay kit. Finally, histological sections of dECM were obtained by standard paraffin embedding. The sections were stained with hematoxylin-eosin (H\&E), Sirius red, and Miller staining and scanned using an Olympus VS120-L100 microscope slide scanner to verify the presence of collagen and elastin.

\subsection{Hydrogel preparation}

To produce the compound dECM-fibrin hydrogel, we first prepare a pre-gel solution. For this, we mix $100 \mu 1$ of $50 \mathrm{mg} / \mathrm{mL}$ dECM stock solution (as described above), $528 \mu \mathrm{l}$ of $50 \mathrm{mg} / \mathrm{mL}$ fibrinogen (Sigma, F3879) solution, 100 $\mu \mathrm{l}$ of HEPES (4-(2-hydroxyethyl)-1-piperazineethanesulfonic acid) $1 \mathrm{M} \mathrm{pH} 7.4$ and $269 \mu 1$ of DMEM. To induce gelling of the pre-gel solution, we then add $1.7 \mu 1$ of Thrombin (Sigma, T1063, 250U/mL) and $1.3 \mu 1$ of calcium chloride $1 \mathrm{M}$, and start incubation at 37 . This yields a final composite gel with $5 \mathrm{mg} / \mathrm{mL} \mathrm{dECM}$ and $26.4 \mathrm{mg} / \mathrm{mL}$ fibrinogen.

Matrigel (Sigma, E1270, solution supplied at $9 \mathrm{mg} / \mathrm{mL}$ ) was diluted to $3 \mathrm{mg} / \mathrm{mL}$ before gelation by using DMEM. Collagen I (Sigma, C4243, solution supplied at $3 \mathrm{mg} / \mathrm{mL}$ ) was diluted to $1 \mathrm{mg} / \mathrm{mL}$ with DMEM prior to gelation. This also neutralized the $\mathrm{pH}$. Finally, the fibrin-collagen I composite was prepared by mixing $157 \mu 1$ of $3 \mathrm{mg} / \mathrm{mL}$ collagen I stock solution, $314 \mu \mathrm{l}$ of DMEM, followed by $528 \mu \mathrm{l}$ of $50 \mathrm{mg} / \mathrm{mL}$ fibrinogen. Gelling was then induced by adding $1.3 \mu \mathrm{l}$ of calcium chloride and 1.7 microliters of thrombin $(250 \mathrm{U} / \mathrm{mL})$.

To include cells, the necessary amount of cells to achieve a final total cell concentration of $10^{6}$ cells $/ \mathrm{mL}$ was pelleted, followed by complete removal of the supernatant. The pellet was resuspended in pre-gel, followed by induction of gelation by addition of calcium chloride and thrombin solutions (fibrin-based hydrogels), and placement at 37 in $5 \% \mathrm{CO}_{2}$ atmosphere.

\subsection{Mechanical properties}

To measure the mechanical properties, fibrin and dECM-fibrin hydrogels were prepared and pipetted into cryovials where they remained for $30 \mathrm{~min}$ at $37^{\circ} \mathrm{C}$ to gel. The samples were then removed from the cryovials and subjected to 
compression testing using a dynamic mechanical analyzer (TA Instruments DMA Q800). The storage and loss moduli are defined as follows[42]:

Storage: $\mathrm{E}^{\prime}=\frac{\sigma_{0}}{\varepsilon_{0}} \cos \delta, \quad$ Loss: $\mathrm{E}^{\prime \prime}=\frac{\sigma_{0}}{\varepsilon_{0}} \sin \delta$

Where $\sigma_{0}$ is stress, $\varepsilon_{0}$ is strain, and $\delta$ is the phase angle or phase lag between the stress and strain.

The Young's modulus is evaluated as: $\quad E=\sqrt{E^{\prime 2}+E^{\prime 2}}$

\subsection{Microstructure characterization}

The microstructure was analyzed by scanning electron microscopy (SEM). For this, the hydrogel sample was frozen and lyophilized. The lyophilized sample was coated with a few nanometers $(5-10 \mathrm{~nm})$ of gold prior to performing SEM imaging.

\section{5. dECM stability study with fluorescently labeled dECM}

To investigate the stability of the dECM in our composite hydrogel, we fluorescently labeled the dECM using rhodamine isothiocyanate[43]. For this, we suspended $300 \mathrm{mg}$ dECM powder in $10 \mathrm{~mL}$ of $0.1 \mathrm{M} \mathrm{HCl}$. The pH was adjusted to 10.3 with $0.9 \mathrm{~mL} \mathrm{NaOH} 1 \mathrm{M}$ and $0.3 \mathrm{~mL}$ of $\mathrm{Na}_{2} \mathrm{CO}_{3} 1 \mathrm{M} .6 \mathrm{mg}$ of rhodamine isothiocyanate was dissolved in $10 \mathrm{~mL}$ of isopropanol, and this solution was added to the reaction mix, followed by $10 \mathrm{~mL}$ of distilled water. After overnight incubation, the dECM was precipated and repeatedly washed with excess isopropanol, until a clear washing solution above strongly stained precipitate was obtained. The precipitate was air-dried overnight. dECMfibrin hydrogels were prepared with fluorescently labeled dECM, using identical procedures as for unlabeled dECM. Fluorescent imaging was conducted after 1,7 , and 14 days of incubation in $\mathrm{PBS}$ at $37^{\circ} \mathrm{C}$.

\subsection{In-vitro cell studies}

\subsubsection{H9c2 cell culture}

H9c2 cells were obtained from the European Collection of Authenticated Cell Cultures (ECACC) (Lot\# 17A028). The cells were cultured in DMEM medium supplemented with $10 \%$ fetal bovine serum and 1\% penicillin and streptomycin in $75 \mathrm{~cm}^{2}$ tissue culture flasks at $37^{\circ} \mathrm{C}$ and $5 \% \mathrm{CO}_{2}$ in an incubator. In accordance with supplier instructions, the $\mathrm{H} 9 \mathrm{c} 2$ cells were passaged before reaching $70-80 \%$ confluency to avoid loss of differentiation potential[38]. 


\subsubsection{Nor-10 cell culture}

NOR-10 (ECACC 90112701) cells were obtained from the European Collection of Authenticated Cell Cultures. The cells were cultured in DMEM medium supplemented with $10 \%$ fetal bovine serum and $1 \%$ penicillin and streptomycin in $75 \mathrm{~cm}^{2}$ tissue culture flasks at $37^{\circ} \mathrm{C}$ and $5 \% \mathrm{CO}_{2}$ in an incubator. They were split before reaching $70-80 \%$ confluency according to supplier's notice[44].

\subsubsection{H9c2 differentiation}

As a starting point in our investigation into use of various hydrogels to enhance cardiogenic differentiation of H9c2 cells, we used a known differentiation procedure of H9c2 cells to cardiomyocytes[45]. This procedure implies simultaneous decrease of the serum percentage to $1 \%$ and application of retinoic acid for 5 days, before one to two last days of culture in expansion medium (DMEM with 10\% FBS without addition of retinoic acid)[45]. Using this protocol, we studied co-culture with Nor-10 fibroblasts $(100 / 0,70 / 30,50 / 50,30 / 70,0 / 100$ H9c2:Nor-10 ratios with the total cell density of $10^{6}$ cells $\left./ \mathrm{mL}\right)$ in different concentrations of retinoic acid $(0-2000 \mathrm{nM})$ for enhancement of the cardiogenic differentiation efficiency. With a fixed 30\% H9c2, 70\% Nor-10 ratio and in the absence of retinoic acid, we then screened various hydrogels such as collagen I, matrigel, and the thrombin-coagulated dECM-fibrin hydrogel for their capacity to substitute for retinoic acid in $\mathrm{H} 9 \mathrm{c} 2$ cardiogenic differentiation.

\subsubsection{Cell seeding onto hydrogel surfaces (2D)}

To study the biochemical influence of different hydrogels on cardiomyocyte differentiation in a 2D geometry, we prepared ca. $0.5 \mathrm{~mm}$ high hydrogel blocks in 48 -well plate. For this, we gelled $50 \mu 1$ of dECM-fibrin, collagen I or matrigel in wells of interest. We then applied H9c2 cells mixed with Nor-10 fibroblasts at a ratio of $30 \%$ to $70 \%$ at a density of $2.5 \times 10^{5}$ cells per $\mathrm{cm}^{2}$ on top of the hydrogels. The differentiation protocol was conducted without addition of retinoic acid: 5 days with 1\% FBS in DMEM, followed by 1-2 days of 10\% FBS in DMEM[45].

\subsubsection{Cell seeding in 3D hydrogel}

To assess whether differentiation could be improved in 3D vs. 2D, we mixed dECM-fibrin pre-gel with Nor-10 and H9c2 cells $\left(70 / 30\right.$ ratio, $10^{6}$ cells $\left./ \mathrm{mL}\right)$. Then thrombin and calcium chloride were added to the solution, and $200 \mu 1$ of the solution was rapidly poured in a 48-well plate where the hydrogel solidified in the incubator. Collagen I and 
Matrigel were used as controls. The differentiation protocol was otherwise identical to the differentiation experiments on the 2D hydrogels, with serum reduction only, in the absence of exogenous retinoic acid.

\subsubsection{Immunostaining and 3D imaging}

For immunostaining, cell culture samples were fixed in $4 \%$ of paraformaldehyde (PFA) for $20 \mathrm{~min}$ at room temperature. Then, $0.1 \%$ TritonX-100 was added to permeabilize the cells for $30 \mathrm{~min}$ at room temperature. By incubating cells with phalloidin-Atto 488 (Sigma 49409, 1:50) for $45 \mathrm{~min}$ at $4^{\circ} \mathrm{C}$, actin filaments were made visible in all types of cells [46].

We assessed the differentiation by measuring the fraction of $\mathrm{H} 9 \mathrm{c} 2$ cells positive for troponin $\mathrm{T}$ by immunofluorescence using the T6277 antibody from Sigma. As this antibody detects both cardiac and skeletal muscle troponin $\mathrm{T}$, we also confirmed cardiac differentiation on dECM-fibrin samples and tissue culture plates using an antibody specific to cardiac troponin T (Abcam, ab8295). This data is provided in supplementary figure S1.

For immunofluorescence, the cells were first blocked with $1 \%$ BSA for 1 hour at $37^{\circ} \mathrm{C}$. Troponin $\mathrm{T}$ primary antibody (Sigma, T6277, 1:50) was then added, followed by incubation overnight at $4^{\circ} \mathrm{C}$. Alexa-568 secondary antibody (Sigma, A10037, 1:1000) was added after washing with DPBS (Gibco 2062235) and incubated for 45min at $37^{\circ} \mathrm{C}$, prior to washing with DPBS and staining with 4',6-diamidino-2-phenylindole (DAPI, 1:2000 from 5mg/ml stock solution) for $5 \mathrm{~min}$. DAPI was replaced with DPBS before imaging the cells under a fluorescence microscope. We quantify the differentiation percentage as the surface area covered by cells expressing troponin $\mathrm{T}$, relative to the total area of the fluorescent images, and normalize to the percentage of $\mathrm{H} 9 \mathrm{c} 2$ cells seeded in co-culture.

\subsubsection{Neonatal cardiomyocytes isolation}

To study the effect of the thrombin-coagulated dECM-fibrin hydrogel on primary cardiomyocytes, we isolated primary rat neonatal cardiomyocytes using the Pierce Primary Cardiomyocyte Isolation Kit, according to the manufacturer's instructions[47]. Organ harvesting was performed on donated sacrificed control animals from unrelated experiments, according to license VD 3290 designed for this specific purpose.

\subsubsection{Calcium imaging}


For calcium imaging, we seeded the primary rat cardiomyocytes $\left(2.5 \times 10^{5} \mathrm{cells} / \mathrm{cm}^{2}\right)$ onto hydrogel slabs prepared in 48-well plates ( $50 \mu 1$ of hydrogel per well, as before). Tissue culture controls were prepared by leaving out the hydrogel polymerization step. After 3 days of culture in DMEM with $10 \%$ FBS, the cultures were loaded with $2 \mu \mathrm{M}$ Fluo-4 AM (Sigma, F14217) for $30 \mathrm{~min}$ at $37^{\circ} \mathrm{C}$. Calcium transients were then recorded using fluorescent microscopy at room temperature. Temporal peak detection was based on a custom ImageJ plugin, implementing the publicly available findpeaks function of Octave[48] in Java. The plugin is available as supplementary S2, but also for download at https://github.com/tbgitoo/calciumImaging, along with source code and a user manual. We used this plugin to evaluate local beating frequency and temporal phase shift from the calcium imaging videos.

\subsubsection{Beating characteristics}

To assess the contractile properties of primary cardiomyocytes in $3 \mathrm{D}$ cultures, we suspended the primary rat cardiomyocytes at a cell density of $10^{6}$ cells $/ \mathrm{mL}$ in pre-gel mixtures of dECM-fibrin, fibrin, and fibrin-collagen I composite. After gelling of $200 \mu \mathrm{L}$ of cell-hydrogel mixture per well in a 48 -well plate, the cultures were followed both visually and videographically. Synchrony and onset of beating was judged visually on a per-well basis. To quantify the mechanical beating rate of the cells, movies were acquired by connecting a video camera to the microscope, while maintaining the samples at $37^{\circ} \mathrm{C}$ in a temperature-controlled chamber. For quantification of the beating frequency, we used the Pulse software (Cellogy Inc.)[49].

\subsection{Statistical analysis}

Data were compared using unpaired t-test (two-tailed, equal variances) in the GraphPad software. Error bars represent the mean \pm standard deviation (SD) of the measurements $(* \mathrm{p}<0.05, * * \mathrm{p}<0.01$, and $* * * \mathrm{p}<0.001)$.

\section{Results}

3.1. Development and characterization of the hybrid hydrogel

\subsubsection{Decellularization characteristics}

We successfully decullarized porcine cardiac extracellular matrix. As shown in Fig. 1A, the total amount of remaining DNA is less than $50 \mathrm{ng} / \mathrm{mg}$ of tissue, indicating essentially complete removal of cells[50]. The concentration of the extracellular matrix components collagen and glycosaminoglycans (GAG) was measured in he native tissue and after decellularization, and shows no loss of these components in the resulting dECM (Fig. 1B, 
1C). H\&E, Sirius red for collagen and Miller for elastin staining confirmed the absence of cells and cell debris and the presence of collagen and elastin in the matrix after decellularization (Fig. 1D).

\subsubsection{Mechanical properties}

Fig. 2A shows the relation between the elastic modulus and fibrinogen concentration in pure fibrin gels. In agreement with literature[12], higher fibrinogen concentrations are associated with higher Young moduli, although at the highest concentrations, a saturation effect can be seen. The evaluation of the storage and loss moduli in dECM-fibrin gels in a linear temperature scan is presented in Fig. 2B.

At 37 , the Young's modulus of the dECM-fibrin hydrogel stabilizes at about $21 \mathrm{kPa}$, falling into the reported range of native adult myocardium from 11.9 to $46.2 \mathrm{kPa}[8]$. This confirms that mechanical properties adequate for heart cell culture can be achieved with the thrombin coagulation method chosen, eliminating the need for less specific crosslinking agents[8], [51].

\subsubsection{Gelation time}

Fig. 2C shows that the gelation time of dECM alone, in the absence of cells, is about 10 minutes. By adding fibrinogen and thrombin to the hydrogel, the gelation time is reduced to about 1-2 minutes, which is sufficient for handling the cells in 3D while avoiding cell sedimentation[27].

\subsubsection{Structural characterization and dECM stability}

Fig. 3A shows a scanning electron micrography image of the dECM-Fibrin hydrogel, whereas Fig. 3B shows specifically the spatial distribution of rhodamine-labelled dECM within the dECM-fibrin composite. The heterogeneous structure of the hydrogel can be observed in both imaging modes. This implies that coherent pieces of dECM subsist and are embedded into fibrin, rather than forming a spatially homogeneous mixture, with heterogeneity probably defining local niche structures. Fig. 3C and 3D indicate stable maintenance of dECM within the dECM-fibrin composite at 7 and 14 days, with no loss of dECM detected. The confocal stack of control dECMfibrin hydrogel without rhodamine labeling shown in Fig. 3E validates the specific detection of rhodamine-labelled dECM as it shows virtual absence of autofluorescence at the exposure settings used for Fig. 3B-3D.

3.2. $\mathrm{H} 9 \mathrm{c} 2$ in co-culture with fibroblast cells 
To investigate the role of extracellular matrix components, and particularly dECM-fibrin as compared to control matrices such as collagen and matrigel, we used cardiogenic differentiation of the H9c2 myoblast line as a model system[52]. The differentiation of $\mathrm{H} 9 \mathrm{c} 2$ cells to cardiomyocytes is traditionally initiated by reducing the serum in the culture medium to $1 \%$ in the presence of all-trans-retinoic acid[45].

In preliminary trials to adapt this procedure for use with our hydrogel systems, we found that retinoic acid induces fibrinolysis. This led to dissolution of fibrin-based gels. Retinoic acid is a known transcriptional activator for the expression of fibrinolytic enzymes[53][54]. In addition, we find the dissolution effect to partly persist even in cellfree systems, indicating a chemical effect as well. Hence, our first step is to optimize cardiac differentiation in H9c2 cells in the absence of retinoids.

Fibroblast-derived matrix has been shown to enhance retinoid-based differentiation of H9c2 cells[39]. Here, we assess whether a similar favorable effect on the H9c2 cardiogenic differentiation can be obtained by direct coculture with Nor-10 fibroblasts[55].

We first optimize the co-culture ratio of $\mathrm{H} 9 \mathrm{c} 2$ cells to Nor-10 fibroblasts. The aim is to increase cardiogenic differentiation of $\mathrm{H} 9 \mathrm{c} 2$ cells in the presence of a minimal amount of retinoic acid. For the sake of simplicity, we investigate this in 2D co-culture, without hydrogel, but systematically vary both the H9c2:Nor-10 seeding ratio and retinoic acid concentrations.

Table 1 shows the percentage of cardiogenically differentiated H9c2 cells, evaluated as the relative surface area covered by cells expressing troponin T corrected for the proportion of H9c2 seeded. Confirmation of specific cardiac troponin $\mathrm{T}$ expression is further given in Supplementary Figure S1B. The heatmap (Table 1A), outlines the differentiation percentage of the $\mathrm{H} 9 \mathrm{c} 2$ cells thus calculated for different values of the ratio of fibroblast cells to H9c2 cells and different concentrations of retinoic acid after 7 days in culture. The results demonstrate that the lower the fraction of $\mathrm{H} 9 \mathrm{c} 2$ seeded, the higher the relative differentiation efficiency, indicating that neighboring Nor10 cells help drive the cardiac differentiation of $\mathrm{H} 9 \mathrm{c} 2$ cells. As a compromise between high relative differentiation efficiency and achievable absolute numbers of differentiated cells, we choose the seeding ratio of $30 \%$ H9c 2 cells to $70 \%$ fibroblast cells as the preferred condition for subsequent experiments. Of note, even this optimized co-culture could not completely substitute for the addition of retinoic acid, as below $62.5 \mathrm{nM}$ the differentiation efficiency started dropping, to reach only about $7 \%$ in the absence of the retinoid (Table $1 \mathrm{~B}$ ). Overall, we retain that performing co- 
culture with fibroblasts improves the differentiation efficiency and dramatically reduces the required concentration of retinoic acid for differentiation.

\subsection{Cell seeding and differentiation in $2 \mathrm{D}$ on hydrogel surfaces}

We next assessed the capacity of various hydrogels to enhance cardiogenic differentiation in H9c2 cells. We also aimed at replacing the retinoic acid in the H9c2 differentiation protocol by similar favorable effects mediated by the microenvironment.

Having confirmed that Nor-10 fibroblasts favor cardiogenic differentiation of H9c2 cells, we seeded H9c2 cells and Nor-10 fibroblasts at a ratio of 30\%:70\% onto hydrogel slabs in 48 well plates. Fig. 4A to $4 \mathrm{D}$ show the extent of troponin $\mathrm{T}$ expression on dECM-fibrin, matrigel, collagen and tissue culture plate control after 1 week of differentiation (confirmation for specific cardiac troponin $\mathrm{T}$ expression in Supplementary Figure S1C). Fig. 4E quantifies the percentage of differentiation of $\mathrm{H} 9 \mathrm{c} 2$ in co-culture with fibroblasts on the different materials. The proportion of differentiated $\mathrm{H} 9 \mathrm{c} 2$ cells is larger on the dECM-fibrin hydrogel than on cell culture plates $(\mathrm{P}=0.0001)$ and matrigel $(\mathrm{P}=0.0024)$, but similar to the one on collagen $(\mathrm{P}=0.645)$. These results suggest that the influence of dECM on the differentiation of $\mathrm{H} 9 \mathrm{c} 2$ cells is likely mediated by its collagen content.

The effect of suitable extracellular matrix is striking: The combination of co-culturing the H9c2 cells with Nor-10 fibroblasts and the presence of either dECM-fibrin or collagen outperforms even the highest concentrations of retinoic acid in $\mathrm{H} 9 \mathrm{c} 2$ monoculture in $2 \mathrm{D}$ (comparison to Table $1 \mathrm{~B}, 100 / 0$ ratio, 2000nM retinoic acid, $\mathrm{P}=0.0059$ between $\mathrm{dECM}$-fibrin and $2 \mathrm{D}$, and $\mathrm{P}=0.0106$ between collagen and $2 \mathrm{D}$ ).

\subsection{Cell seeding and differentiation in $3 \mathrm{D}$ hydrogels}

Having evaluated and optimized differentiation efficiency on the surface of various hydrogels, our next step was to proceed to a truly 3D co-culture configuration. For this, we included the suspended cells H9c2 and Nor-10 cells to be co-cultured in the dECM-fibrin hydrogel prior to gel formation. Cells cultured in the 3D structure of the dECMfibrin hydrogel attached, spread, and formed a network throughout the wells. We also attempted 3D culture in collagen I and matrigel[56], [57], but could not carry out the entire differentiation protocol in 3D, since after about 2 days, the gels had become very soft with a large part of the cells attached to the floor of the wells. 
Fig. 5 shows the H9c2 cell differentiation in the 3D hydrogel using Troponin T staining. The cells attached, spread, and formed a network throughout the wells. The differentiation percentage of H9c2 cells in co-culture with fibroblasts in 3D dECM-fibrin hydrogel was $83 \pm 8 \%$. This is significantly better than the 2D co-cultures of H9c2 and fibroblast cells on top of dECM-fibrin coated surfaces $(P=0.0007)$, indicating a specific advantage of the 3D configuration. To achieve a similar differentiation efficiency in $2 \mathrm{D}$, the combination of retinoic acid and co-culture is required $(\mathrm{P}=0.77)$.

Due to the fragile nature of the pure collagen I gels, we could not assess whether there is a difference between dECM-fibrin and collagen I regarding H9c2 differentiation in 3D. However, from the 2D results given in Fig. 4E, it seems that the primary advantage of the dECM-fibrin gel over collagen I in the H9c2 system is mechanical ruggedness, both gels showing high differentiation capacity.

Overall, we conclude that the very high differentiation efficiency in our 3D co-culture system affords the freedom to avoid retinoids altogether. Co-culture with Nor-10 fibroblasts, the $3 \mathrm{D}$ environment, and a collagen-based matrix all have a positive effect on cardiac differentiation of $\mathrm{H} 9 \mathrm{c} 2$ cells.

\subsection{Neonatal cardiac cells on $2 \mathrm{D}$ and in $3 \mathrm{D}$ hydrogels}

To study the effect of different hydrogels with a more physiologically relevant cell system, we next cultured primary neonatal cardiomyocytes on surfaces (2D culture) of dECM-fibrin hydrogel, in comparison with various controls: tissue culture plates, the commercial matrices collagen I and matrigel, as well as a fibrin-collagen I composite. This latter is the closest analog to dECM-fibrin with chemically defined composition. To assess electrophysiological activity, we videographically recorded intracellular calcium ionic concentration variations by using the $\mathrm{Ca}^{2+}$ indicator Fluo-4 AM at 3 days of culture. Sample videos are provided as supplementary Videos S4 (dECM-fibrin), S5 (fibrin-collagen I), S6 (collagen I), S7 (Matrigel), S8 (tissue culture plate). From the calcium oscillation videos, we evaluated local frequency (Fig. 6A-6E) and local phase (Fig. 6F-6J). The results indicate essentially perfect synchrony on the dECM-fibrin hydrogel, regarding both frequency (6E) and phase (6J). This is followed by fibrincollagen I where the phase analysis (6I) shows some individual desynchronized cells and regions with increased lag. The other conditions show even higher degrees of variability in phase and frequency, common timing being nearly lost on matrigel (6G). This indicates that dECM-fibrin performs best regarding synchronization of calcium influx. The result correlates with morphological observation: cell spread and morphological connectivity improve in 
parallel with synchrony along the series matrigel, collagen I and tissue culture plates, fibrin-collagen I, culminating in dECM-fibrin hydrogels.

Having established optimal properties of the dECM-fibrin matrix regarding synchronization of calcium influx, we next investigated mechanical contraction activity. In light of the results obtained by calcium imaging, we compared $3 \mathrm{D}$ cultures of rat neonatal cardiomyocytes in dECM-fibrin hydrogels to similar cultures in fibrin alone and fibrincollagen I.

The normal beating rate of the neonatal rat heart is around 276-423 beats per minute (bpm)[58], whereas for isolated primary cells in-vitro on standard cell culture conditions it is about 100-115 bpm, (manufacturer's notice[59], confirmed in preliminary trials). Fig. $6 \mathrm{~K}$ shows a profound effect of the extracellular matrix environment on the beating rate. At $77 \mathrm{bpm}$ at 5 days of culture, pure fibrin gels sustain a relatively low beating rate, whereas fibrincollagen I (166bpm, $\mathrm{P}=0.0001$ vs. fibrin) and even more so dECM-fibrin cultures (206bpm, $\mathrm{P}=0.0001$ vs. fibrincollagen I) approach the expected rate of the neonatal heart. The recovery of the mechanical beating function was gradual as indicated by the progressive rise of beating frequency for all conditions in Fig. $6 \mathrm{~K}$, and started earlier in the dECM-fibrin hydrogel as compared to the other conditions.

Finally, in Fig. 6L, we visually quantified the synchrony of the neonatal cardiac cell cultures. The neonatal cardiomyocyte extracts seeded in dECM-fibrin gel displayed synchronous contractions from the first day on, and for at least 10 days (Supplementary video S3). In contrast, cardiomyocytes seeded in fibrin did not show synchronous beating until day 5 and typically stopped beating after 7 days. The cardiomyocytes cultured in fibrin-collagen I showed intermediate behavior by reaching synchronicity at day 3 , but similar to fibrin, they did not beat after 7 days. These results confirm the superior results obtained with the dECM-fibrin hydrogel in calcium imaging (Fig. 6A-6J), and make dECM-fibrin the optimal hydrogel for primary neonatal cardiomyocyte culture among the various options compared here.

\section{Discussion}

In this study, we develop a hydrogel blend of fibrin and decellularized porcine cardiac matrix to improve the cellular niche for cardiomyocyte cell culture. For this, we successfully decellularized porcine ventricular tissue, with maintenance of collagen, glycosaminoglycanes and elastin components. The addition of fibrinogen to the dECM 
thus obtained, followed by coagulation with thrombin, provided a Young modulus of about 20kPa, in the range of adult cardiac tissue[8]. We used this system in conjunction with fibroblast co-culture to robustly differentiate the H9c2 myoblast line into a cardiomyocyte phenotype while avoiding retinoic acid. Finally, we demonstrated excellent maintenance of electromechanical activity in neonatal cardiomyocytes by quantification of beating activity as well as calcium imaging.

An important aim in the design of the hydrogel system was to match the physical stiffness of native myocardium (10-50kPa range, [60][8]). To mechanically reinforce the intrinsically soft cardiac dECM, we chose the specific coagulation of fibrinogen by thrombin, as opposed to more generic agents such as transglutaminase[30][61] or genipin[39][62] We find the thrombin-coagulated fibrin gels to be softer than similar gels crosslinked by transglutaminase[30], but we could compensate for this by increasing the fibrinogen concentration correspondingly. The specificity of thrombin[63] is advantageous for both in-vitro and anticipated in-vivo applications, and probably allowed us to avoid cellular toxicity observed in transglutaminase crosslinking[30].

We used the $\mathrm{H} 9 \mathrm{c} 2$ cell line to investigate cardiogenic differentiation. By combining 3D embedding into dECM fibrin hydrogels with co-culture with Nor-10 fibroblasts, we obtained over $80 \%$ differentiation efficiency. While the utility of adding fibroblasts or fibroblast-derived matrix in $\mathrm{H} 9 \mathrm{c} 2$ differentiation has repeatedly been demonstrated[64][29][60], the differentiation efficiency in our system is rather high compared to previous reports[64][39]. Nota bene, we obtain our results in the absence of retinoids. We achieved this by stepwise optimization of three key elements of the cardiac cell environment: choice of the extracellular matrix, its physical properties, and the co-culture composition. The result should simplify pharmacological screening, as retinoids are known to broadly affect cellular processes beyond specific cardiac differentiation[66]. Further, avoiding the strong transcriptional effects of retinoids[66] could also simplify investigation of communication between cardiac fibroblasts and cardiomyocyte, which today remains only partially understood[67].

The ultimate aim of an in-vitro cardiac model should be to replicate the electromechanical beating function. Comparative culture of freshly isolated rat cardiomyocytes on dECM-fibrin, fibrin-collagen I and fibrin control hydrogels indicates specific advantages of the dECM-fibrin composite as it performs best regarding recovery of physiological beating frequency and reacquisition of synchrony. In line with these results, dECM-fibrin also outperformed collagen I, matrigel, tissue culture plates, and also fibrin-collagen I regarding synchronization in 
calcium imaging. The result correlates well with morphological analysis: cell spreading and enhanced geometric contacts are overall associated with higher frequency and better synchrony. Given that we seed a native cardiomyocyte population including various pacemaker cells, these results replicate native cardiac physiology[68]: better connectivity directly provides better synchrony, but also better spread of the highest pacemaker frequency. This subtly graded response of the native cardiomyocyte population contrasts with the differentiation of the H9c2 cell line, where the presence of collagen was as efficient as the dECM-fibrin composite in inducing cardiogenic differentiation.

Interaction of cardiomyocytes with extracellular matrix proteins is known to be of prime importance for cardiac differentiation and electrophysiological maintenance. For example, a multitude of integrins with affinity for collagen I, but also laminin and fibronectin are expressed on cardiomyocytes[69][70][71][72]. It is fully conceivable that the presence of a more complete mixture of extracellular matrix components in the cardiac dECM is important not only for fine electrophysiological regulation, but also for cell spreading and thus geometrical connectivity of neonatal cardiomyocytes. In contrast, collagen I may suffice in $\mathrm{H} 9 \mathrm{c} 2$ to induce a pro-cardiogenic, but ultimately nonfunctional cell state.

Mechanical aspects probably play a more important role in mechanically beating cells as well. Ideal energy transmission to the substrate is expected to occur at near physiological stiffness (neither too soft, preventing force development, nor too stiff, preventing substrate deformation)[73]-[75]. Mechanical feedback[71], could therefore help explain why the most physiological frequency and best synchrony for the neonatal cardiomyocytes are indeed found for the composite hydrogels, and not for the softer collagen, matrigel or pure fibrin or on the other hand the hard tissue culture plates.

\section{Conclusion}

In this work, we demonstrate a new combination of two natural hydrogels for myocardium regeneration: $\mathrm{dECM}$ from porcine ventricular heart tissue, admixed with fibrinogen and coagulated under cell-compatible conditions with thrombin. The mechanical properties are adjusted to match the ones of the native heart tissue, while the choice of thrombin as a specific crosslinking agent eliminates concerns of toxicity with more generic agents. We successfully test the system in the context of highly efficient, retinoid-free cardiac differentiation of the myoblast line H9c2. In neonatal cardiomyocytes, we show enhanced recovery, synchrony and beating frequency as compared to various 
controls, demonstrating specific advantageous of using the dECM-fibrin hydrogel over collagen analogs, fibrin alone, matrigel and tissue culture plates. We anticipate use of this system both as robust cardiac 3D cell model for drug screening, and as a building block for $3 \mathrm{D}$ printing, tissue engineering and transplantation in regenerative medicine.

\section{Acknowledgements}

The research presented in this paper is supported by the Swiss Government Excellence Scholarship ESKAS-Nr: 2015.1050, and the SNSF Professorship Grant PP00P2_163684. The authors thank Prof. Lashuel's lab, especially Elena Gasparotto for providing neonatal hearts and Prof. Rohr's lab, especially Regula Fluckiger Labrada for providing isolated neonatal cardiac cells, Dr. Marisa Jaconi for providing advice on cardiac cell biology and Fabien Bonini for scanning the histology slides. We also thank Arnaud Bertsch for his priceless support. Special thanks of gratitude go to Marina Braschler and Sara Ancel. Further, we acknowledge the services of the Bioimaging and Optics Platform (BIOP), Center of MicroNanoTechnology(CMi) and Interdisciplinary Centre for Electron Microscopy(CIME) at EPFL, and the Bioimaging Core Facility of the Faculty of Medicine of the University of Geneva.

7. Declaration of conflicts of interest

T. Braschler and P. Renaud declare financial interests in Volumina-Medical SA, Lausanne, Switzerland. The other authors declare no conflicts of interest.

\section{Supplementaries}

Supplementary files and raw research data are available on the Zenodo repository :

https://zenodo.org/record/3556793\#.XeAxJ7971R0

S1: Staining for cardiac Troponin $\mathrm{T}$

S2: calciumImaging_.jar. Source code, documentation and user manual at https://github.com/tbgitoo/calciumImaging

S3: Beating motion of rat neonatal cardiomyocytes in dECM-fibrin 
S4: calcium imaging: video of calcium oscillations in rat neonatal cardiomyocytes on dECM-fibrin

S5: calcium imaging: video of calcium oscillations in rat neonatal cardiomyocytes on fibrin-collagen I

S6: calcium imaging: video of calcium oscillations in rat neonatal cardiomyocytes on collagen I

S7: calcium imaging: video of calcium oscillations in rat neonatal cardiomyocytes on matrigel

S8: calcium imaging: video of calcium oscillations in rat neonatal cardiomyocytes on tissue culture plastic

9. References

[1] World Health Organization, "Cardiovascular diseases (CVDs)," May-2017. [Online]. Available: https://www.who.int/en/news-room/fact-sheets/detail/cardiovascular-diseases-(cvds). [Accessed: 29-Nov-2019].

[2] T. Kalogeris, C. P. Baines, M. Krenz, and R. J. Korthuis, "Ischemia/Reperfusion," Compr Physiol, vol. 7, no. 1, pp. 113-170, Dec. 2016.

[3] W. Richardson, S. Clarke, T. Quinn, and J. Holmes, "Physiological Implications of Myocardial Scar Structure,” Compr Physiol, vol. 5, no. 4, pp. 1877-1909, Sep. 2015.

[4] G. Camci-Unal, N. Annabi, M. R. Dokmeci, R. Liao, and A. Khademhosseini, "Hydrogels for cardiac tissue engineering," NPG Asia Materials, vol. 6, no. 5, p. e99, May 2014.

[5] L. Hortells, A. K. Z. Johansen, and K. E. Yutzey, "Cardiac Fibroblasts and the Extracellular Matrix in Regenerative and Nonregenerative Hearts," J Cardiovasc Dev Dis, vol. 6, no. 3, Aug. 2019.

[6] P. Bajaj, X. Tang, T. A. Saif, and R. Bashir, "Stiffness of the substrate influences the phenotype of embryonic chicken cardiac myocytes," Journal of Biomedical Materials Research Part A, vol. 95A, no. 4, pp. 1261$1269,2010$.

[7] G. Agmon and K. L. Christman, "Controlling stem cell behavior with decellularized extracellular matrix scaffolds," Current Opinion in Solid State \& Materials Science, vol. 20, no. 4, pp. 193-201, 2016.

[8] B. Bhana et al., "Influence of substrate stiffness on the phenotype of heart cells," Biotechnology and Bioengineering, vol. 105, no. 6, pp. 1148-1160.

[9] T. A. Baudino, W. Carver, W. Giles, and T. K. Borg, "Cardiac fibroblasts: friend or foe?," American Journal of Physiology-Heart and Circulatory Physiology, vol. 291, no. 3, pp. H1015-H1026, Sep. 2006.

[10] J. M. Singelyn, J. A. DeQuach, S. B. Seif-Naraghi, R. B. Littlefield, P. J. Schup-Magoffin, and K. L. Christman, "Naturally derived myocardial matrix as an injectable scaffold for cardiac tissue engineering,"

Biomaterials, vol. 30, no. 29, pp. 5409-5416, Oct. 2009.

[11] S. Canonico, "The use of human fibrin glue in the surgical operations," Acta Biomed, vol. 74 Suppl 2, pp. 21-25, 2003.

[12] J. Brouwers, "Influence of fibrinogen concentration on the Young's modulus in fibrin gels," Master Thesis, Eindhoven, 2002.

[13] T. D. Johnson et al., "Human versus porcine tissue sourcing for an injectable myocardial matrix hydrogel," Biomater. Sci., vol. 2, no. 5, pp. 735-744, 2014.

[14] S. B. Seif-Naraghi et al., "Safety and Efficacy of an Injectable Extracellular Matrix Hydrogel for Treating Myocardial Infarction," Science Translational Medicine, vol. 5, no. 173, pp. 173ra25-173ra25, Feb. 2013.

[15] F. Gattazzo, A. Urciuolo, and P. Bonaldo, "Extracellular matrix: A dynamic microenvironment for stem 
cell niche," Biochim Biophys Acta, vol. 1840, no. 8, pp. 2506-2519, Aug. 2014.

[16] M. Verhulsel, M. Vignes, S. Descroix, L. Malaquin, D. M. Vignjevic, and J. Viovy, "Biomaterials A review of microfabrication and hydrogel engineering for micro-organs on chips," Biomaterials, vol. 35, no. 6, pp. 1816-1832, 2014.

[17] “2015 4th TERMIS World CongressBoston, MassachusettsSeptember 8-11, 2015,” Tissue Engineering Part A, vol. 21, no. S1, p. S-1, Aug. 2015.

[18] L. T. Saldin, M. C. Cramer, S. S. Velankar, L. J. White, and S. F. Badylak, "Extracellular Matrix Hydrogels from Decellularized Tissues: Structure and Function," Acta Biomater, vol. 49, pp. 1-15, Feb. 2017.

[19] N. Annabi et al., "Highly Elastic Micropatterned Hydrogel for Engineering Functional Cardiac Tissue," Adv Funct Mater, vol. 23, no. 39, Oct. 2013.

[20] M. J. A. van Luyn et al., "Cardiac tissue engineering: characteristics of in unison contracting two- and three-dimensional neonatal rat ventricle cell (co)-cultures," Biomaterials, vol. 23, no. 24, pp. 4793-4801, Dec. 2002.

[21] H. Park, B. L. Larson, M. E. Kolewe, G. Vunjak-Novakovic, and L. E. Freed, "Biomimetic scaffold combined with electrical stimulation and growth factor promotes tissue engineered cardiac development," Exp Cell Res, vol. 321, no. 2, pp. 297-306, Feb. 2014.

[22] Y. Xing et al., "Construction of engineered myocardial tissues in vitro with cardiomyocyte-like cells and a polylactic-co-glycolic acid polymer," Mol Med Rep, vol. 20, no. 3, pp. 2403-2409, Sep. 2019.

[23] R. Feiner et al., "Engineered hybrid cardiac patches with multifunctional electronics for online monitoring and regulation of tissue function," Nat Mater, vol. 15, no. 6, pp. 679-685, Jun. 2016.

[24] B. W. Streeter and M. E. Davis, "Therapeutic Cardiac Patches for Repairing the Myocardium," Adv. Exp. Med. Biol., vol. 1144, pp. 1-24, 2019.

[25] Kutschka Ingo et al., "Collagen Matrices Enhance Survival of Transplanted Cardiomyoblasts and Contribute to Functional Improvement of Ischemic Rat Hearts,” Circulation, vol. 114, no. 1_supplement, pp. I-167, Jul. 2006.

[26] L. T. Saldin, M. C. Cramer, S. S. Velankar, L. J. White, and S. F. Badylak, "Acta Biomaterialia Extracellular matrix hydrogels from decellularized tissues : Structure and function," Acta Biomaterialia, vol. 49, pp. $1-15,2017$.

[27] Z. Wang and J. M. Belovich, "A simple apparatus for measuring cell settling velocity," Biotechnology Progress, vol. 26, no. 5, pp. 1361-1366, Sep. 2010.

[28] "Tailoring Material Properties of Cardiac Matrix Hydrogels to Induce Endothelial Differentiation of Human Mesenchymal Stem Cells.” [Online]. Available: https:/www.ncbi.nlm.nih.gov/pmc/articles/PMC4684185/. [Accessed: 02-Oct-2019].

[29] L. G. Bracaglia and J. P. Fisher, "ECM-Based Biohybrid Materials for Engineering Compliant, MatrixDense Tissues," Adv Healthc Mater, vol. 4, no. 16, pp. 2475-2487, Nov. 2015.

[30] C. Williams et al., "Cardiac Extracellular Matrix-Fibrin Hybrid Scaffolds with Tunable Properties for Cardiovascular Tissue Engineering," Acta Biomater, vol. 14, pp. 84-95, Mar. 2015.

[31] "Celiac disease and transglutaminase 2: Model for posttranslational modification of antigens and HLA association in the pathogenesis of autoimmune disorders." [Online]. Available: https://www.ncbi.nlm.nih.gov/pmc/articles/PMC3428143/. [Accessed: 02-Oct-2019].

[32] Y. P. Kong, B. Carrion, R. K. Singh, and A. J. Putnam, "Matrix identity and tractional forces influence indirect cardiac reprogramming," Scientific Reports, vol. 3, no. 1, Dec. 2013.

[33] P. Zhou and W. T. Pu, "Recounting cardiac cellular composition," Circ Res, vol. 118, no. 3, pp. 368-370, Feb. 2016. 
[34] P. Camelliti, T. K. Borg, and P. Kohl, "Structural and functional characterisation of cardiac fibroblasts," Cardiovascular Research, vol. 65, no. 1, pp. 40-51, 2005.

[35] A. L. Mackey, M. Magnan, B. Chazaud, and M. Kjaer, "Human skeletal muscle fibroblasts stimulate in vitro myogenesis and in vivo muscle regeneration," J Physiol, vol. 595, no. 15, pp. 5115-5127, Aug. 2017.

[36] Kane Christopher and Terracciano Cesare M, “Abstract 18700: Cardiac Fibroblast Co-Culture Promotes More Adult-Like Electrophysiological Properties in Human Induced Pluripotent Stem Cell-Derived Cardiomyocytes," Circulation, vol. 134, no. suppl_1, pp. A18700-A18700, Nov. 2016.

[37] B. W. Kimes and B. L. Brandt, "Properties of a clonal muscle cell line from rat heart," Experimental Cell Research, vol. 98, no. 2, pp. 367-381, Mar. 1976.

[38] A. F. Branco, S. P. Pereira, S. Gonzalez, O. Gusev, A. A. Rizvanov, and P. J. Oliveira, "Gene Expression Profiling of H9c2 Myoblast Differentiation towards a Cardiac-Like Phenotype," PLOS ONE, vol. 10, no. 6, p. e0129303, Jun. 2015.

[39] M. Suhaeri et al., "Cardiomyoblast (H9c2) Differentiation on Tunable Extracellular Matrix Microenvironment," Tissue Eng Part A, vol. 21, no. 11-12, pp. 1940-1951, Jun. 2015.

[40] J. E. Balmer and R. Blomhoff, "Gene expression regulation by retinoic acid,” J. Lipid Res., vol. 43, no. 11, pp. 1773-1808, Nov. 2002.

[41] D. Y. Yang, B. Eng, J. S. Waye, J. C. Dudar, and S. R. Saunders, "Improved DNA extraction from ancient bones using silica-based spin columns," American Journal of Physical Anthropology, vol. 105, no. 4, pp. 539-543, 1998.

[42] M. A. Meyers and K. K. Chawla, Mechanical behavior of materials, 2nd ed. Cambridge ; New York: Cambridge University Press, 2009.

[43] A. Béduer et al., "Additive manufacturing of hierarchical injectable scaffolds for tissue engineering," Acta Biomaterialia, vol. 76, pp. 71-79, Aug. 2018.

[44] “detail." [Online]. Available: https://www.pheculturecollections.org.uk/products/celllines/generalcell/detail.jsp?refId=90112701\&collection=ecacc_gc. [Accessed: 28-Oct-2019].

[45] A. F. Branco, S. P. Pereira, S. Gonzalez, O. Gusev, A. A. Rizvanov, and P. J. Oliveira, "Gene Expression Profiling of H9c2 Myoblast Differentiation towards a Cardiac-Like Phenotype,” PLOS ONE, vol. 10, no. 6, p. e0129303, Jun. 2015.

[46] "Phalloidin-Atto 488 49409," Sigma-Aldrich. [Online]. Available:

https://www.sigmaaldrich.com/catalog/product/sigma/49409. [Accessed: 08-Oct-2019].

[47] "Pierce ${ }^{\mathrm{TM}}$ Primary Cardiomyocyte Isolation Kit." [Online]. Available: https:/www.thermofisher.com/order/catalog/product/88281?SID=srch-srp-88281\#/88281?SID=srch-srp-88281. [Accessed: 07-Oct-2019].

[48] "findpeaks.m in octave-signal | source code search engine." [Online]. Available: https://searchcode.com/codesearch/view/64213481/. [Accessed: 13-Nov-2019].

[49] M. Maddah et al., "A Non-invasive Platform for Functional Characterization of Stem-Cell-Derived Cardiomyocytes with Applications in Cardiotoxicity Testing," Stem Cell Reports, vol. 4, no. 4, pp. 621-631, Mar. 2015 .

[50] F. Pati et al., "Printing three-dimensional tissue analogues with decellularized extracellular matrix bioink," Nat Commun, vol. 5, Jun. 2014.

[51] B. Schoen et al., "Electrospun Extracellular Matrix: Paving the Way to Tailor-Made Natural Scaffolds for Cardiac Tissue Regeneration,” Advanced Functional Materials, vol. 27, no. 34, p. 1700427. 
[52] S. J. Watkins, G. M. Borthwick, and H. M. Arthur, "The H9C2 cell line and primary neonatal cardiomyocyte cells show similar hypertrophic responses in vitro," In Vitro Cell.Dev.Biol.-Animal, vol. 47, no. 2, pp. 125-131, Feb. 2011.

[53] A. C. Gidlöf, P. Ocaya, O. Krivospitskaya, and A. Sirsjö, "Vitamin A: a drug for prevention of restenosis/reocclusion after percutaneous coronary intervention?,” Clin. Sci., vol. 114, no. 1, pp. 19-25, Jan. 2008.

[54] M. S. Tallman et al., "Effects of all-trans retinoic acid or chemotherapy on the molecular regulation of systemic blood coagulation and fibrinolysis in patients with acute promyelocytic leukemia," J. Thromb. Haemost., vol. 2, no. 8, pp. 1341-1350, Aug. 2004.

[55] “Cellosaurus cell line NOR-10 (CVCL_3939)." [Online]. Available: https://web.expasy.org/cellosaurus/CVCL_3939. [Accessed: 30-Sep-2019].

[56] M. G. McCoy, J. M. Wei, S. Choi, J. P. Goerger, W. Zipfel, and C. Fischbach, “Collagen Fiber Orientation Regulates 3D Vascular Network Formation and Alignment," ACS Biomater. Sci. Eng., vol. 4, no. 8, pp. 2967-2976, Aug. 2018.

[57] K. Zhang and A. Manninen, "3D Cell Culture Models of Epithelial Tissues," in Kidney Organogenesis: Methods and Protocols, S. Vainio, Ed. New York, NY: Springer New York, 2019, pp. 77-84.

[58] C. M. Zehendner, H. J. Luhmann, and J.-W. Yang, “A Simple and Novel Method to Monitor Breathing and Heart Rate in Awake and Urethane-Anesthetized Newborn Rodents," PLOS ONE, vol. 8, no. 5, p. e62628, May 2013.

[59] "One-Hour Procedure to Isolate Primary Cardiomyocytes from Neonatal Mouse and Rat Hearts - CH." [Online]. Available: https://www.thermofisher.com/uk/en/home/life-science/protein-biology/protein-biologylearning-center/protein-biology-resource-library/protein-biology-application-notes/one-hour-procedure-isolateprimary-cardiomyocytes-neonatal-mouse-rat-hearts.html. [Accessed: 19-Sep-2019].

[60] "Substrate Stiffness Affects the Functional Maturation of Neonatal Rat Ventricular Myocytes," Biophysical Journal, vol. 95, no. 7, pp. 3479-3487, Oct. 2008.

[61] M. T. Gundersen, J. W. Keillor, and J. N. Pelletier, "Microbial transglutaminase displays broad acylacceptor substrate specificity," Appl. Microbiol. Biotechnol., vol. 98, no. 1, pp. 219-230, Jan. 2014.

[62] "Mechanism and kinetics of the crosslinking reaction between biopolymers containing primary amine groups and genipin - Butler - 2003 - Journal of Polymer Science Part A: Polymer Chemistry - Wiley Online Library." [Online]. Available: https://onlinelibrary.wiley.com/doi/10.1002/pola.10960. [Accessed: 07-Oct-2019].

[63] M. Gallwitz, M. Enoksson, M. Thorpe, and L. Hellman, "The Extended Cleavage Specificity of Human Thrombin," PLOS ONE, vol. 7, no. 2, p. e31756, Feb. 2012.

[64] M. Suhaeri et al., "Novel Platform of Cardiomyocyte Culture and Coculture via Fibroblast-Derived MatrixCoupled Aligned Electrospun Nanofiber," 20-Dec-2016. [Online]. Available: https://pubs.acs.org/doi/full/10.1021/acsami.6b14020. [Accessed: 07-Oct-2019].

[65] R. J. Bick, M. B. Snuggs, B. J. Poindexter, L. M. Buja, and W. B. V. Winkle, "Physical, contractile and calcium handling properties of neonatal cardiac myocytes cultured on different matrices," Cell Adhesion and Communication, vol. 6, no. 4, pp. 301-310, Jan. 1998.

[66] F.-J. Huang et al., "Adverse effects of retinoic acid on embryo development and the selective expression of retinoic acid receptors in mouse blastocysts," Hum Reprod, vol. 21, no. 1, pp. 202-209, Jan. 2006.

[67] J. Pellman, J. Zhang, and F. Sheikh, "Myocyte-Fibroblast Communication in Cardiac Fibrosis and Arrhythmias: Mechanisms and Model Systems," J Mol Cell Cardiol, vol. 94, pp. 22-31, May 2016.

[68] H. C. Pape et al., Physiologie. Stuttgart: Georg Thieme Verlag, 2018.

[69] B. Liu et al., "Role of Cyclic Strain Frequency in Regulating the Alignment of Vascular Smooth Muscle Cells In Vitro,” Biophysical Journal, vol. 94, no. 4, pp. 1497-1507, Feb. 2008. 
[70] S. Israeli-Rosenberg, A. M. Manso, H. Okada, and R. S. Ross, "Integrins and Integrin-Associated Proteins in the Cardiac Myocyte," Circ Res, vol. 114, no. 3, pp. 572-586, Jan. 2014.

[71] O. Cohen and S. A. Safran, "Theory of frequency response of mechanically driven cardiomyocytes," Sci Rep, vol. 8, no. 1, pp. 1-8, Feb. 2018.

[72] Ross Robert S. and Borg Thomas K., "Integrins and the Myocardium," Circulation Research, vol. 88, no. 11, pp. 1112-1119, Jun. 2001.

[73] "Heart-Specific Stiffening in Early Embryos Parallels Matrix and Myosin Expression to Optimize Beating ScienceDirect." [Online]. Available:

https://www.sciencedirect.com/science/article/pii/S0960982213013328?via\%3Dihub. [Accessed: 13-Nov-2019].

[74] "Embryonic cardiomyocytes beat best on a matrix with heart-like elasticity: scar-like rigidity inhibits beating." [Online]. Available: https://www.ncbi.nlm.nih.gov/pmc/articles/PMC2740334/. [Accessed: 13-Nov-2019].

[75] "Mechanical communication in cardiac cell synchronized beating | Nature Physics." [Online]. Available: https://www.nature.com/articles/nphys3619?proof=t\&draft=journal. [Accessed: 13-Nov-2019].

Figures and captions:
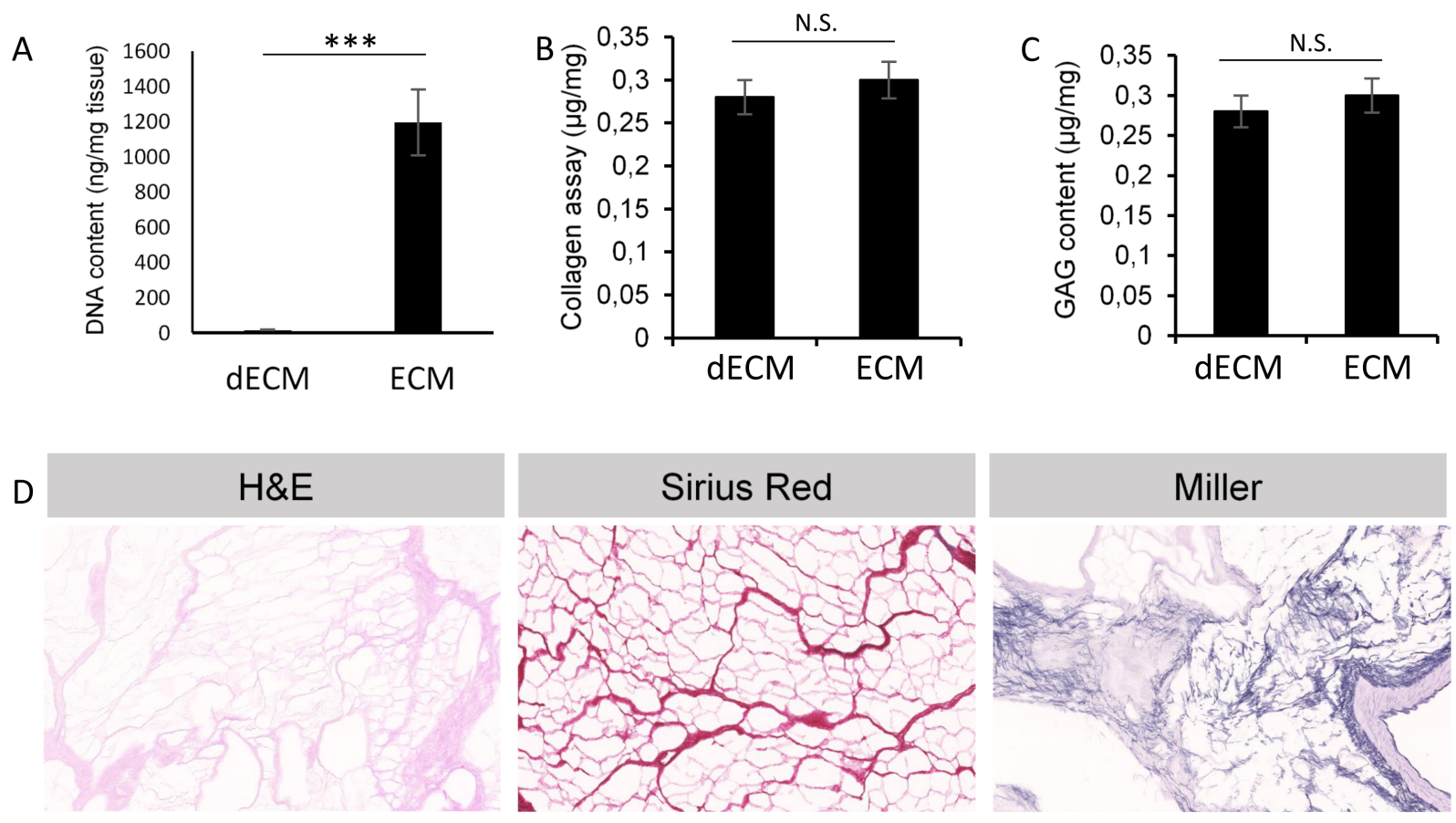

Figure 1 dECM characterization. A) DNA, B) collagen, and C) Glycosaminoglycane (GAG) content of the dECM. D) H\&E, Sirius red and Miller staining used for staining the nuclei, collagen and elastin respectively, which proves the presence of collagen and elastin and removal of the DNA. ${ }^{* * *}=$ significant difference for $p<0.001$ ( $N=3-4$ per condition). Scale bar: $100 \mu m$. 
bioRxiv preprint doi: https://doi.org/10.1101/2020.01.30.927319; this version posted January 31, 2020. The copyright holder for this preprint (which was not certified by peer review) is the author/funder. All rights reserved. No reuse allowed without permission.

A

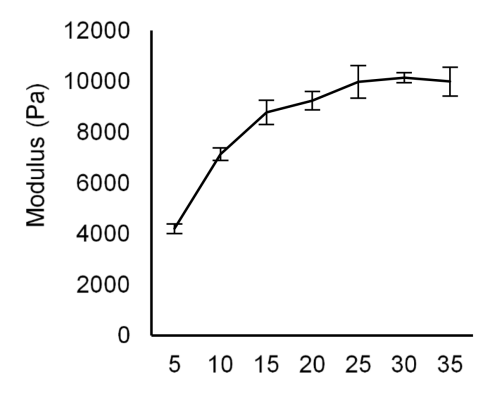

Fibrinogen concentration $(\mathrm{mg} / \mathrm{ml})$
B

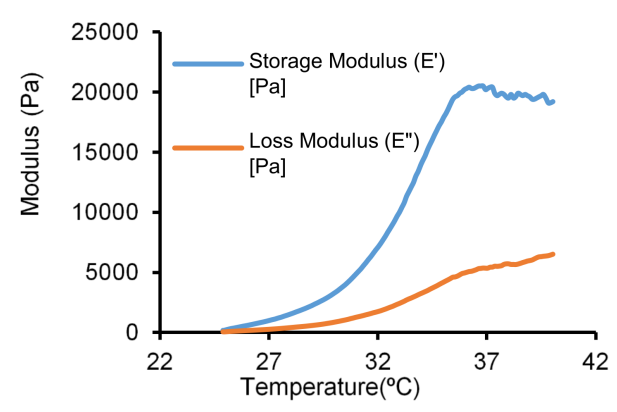

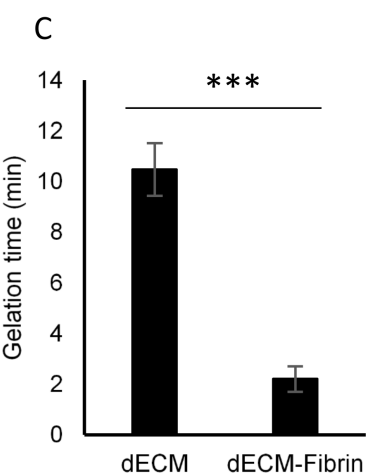

Figure 2 Mechanical characterization. A) Stiffness of fibrin gels at different concentrations at room temperature. This allows to determine the optimum concentration of fibrinogen in the hydrogel. B) Storage and loss moduli of the dECM-fibrin hydrogel in a temperature scan (from 25 to 40). The Young's modulus of the hydrogel can be calculated from these measurements. It is comparable to the one of the native heart. C) Gelation time of the dECM and dECM-Fibrin hydrogels at 37 . In the case of the dECM-Fibrin hydrogel, 2 minutes are sufficient for handling the gel *** = significant difference, $p<0.001$ ( $N=3-4$ per condition).
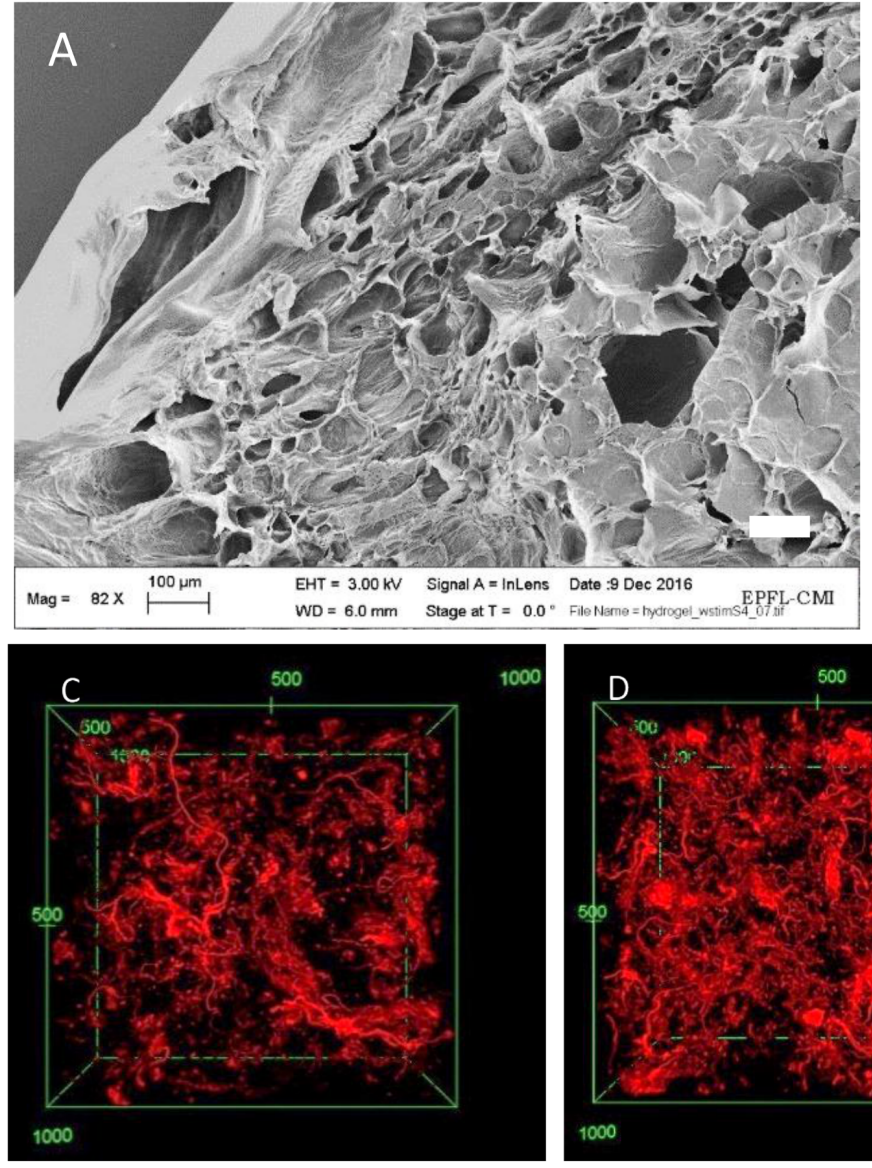
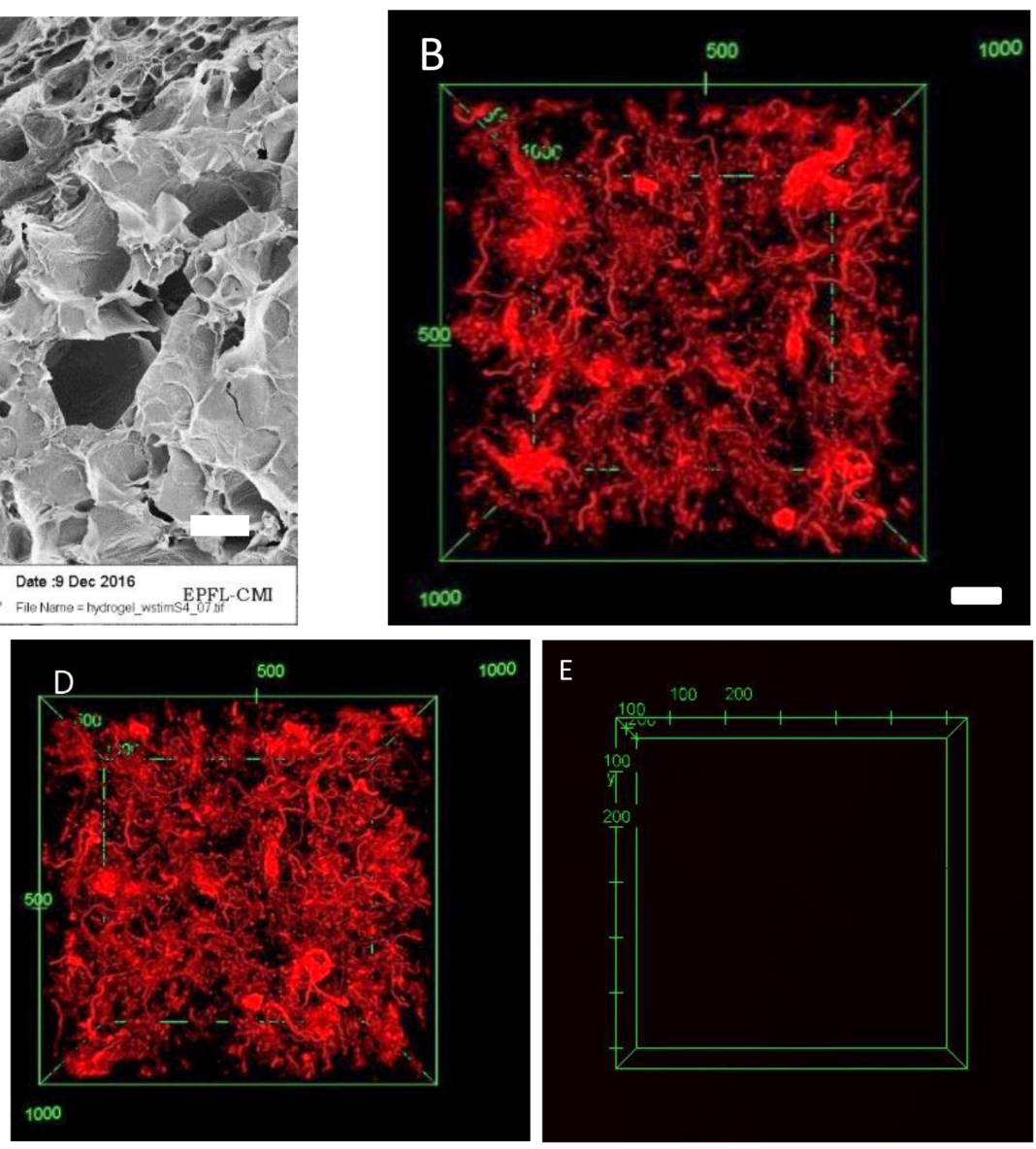
bioRxiv preprint doi: https://doi.org/10.1101/2020.01.30.927319; this version posted January 31, 2020. The copyright holder for this preprint (which was not certified by peer review) is the author/funder. All rights reserved. No reuse allowed without permission.

Figure 3. Microstructural analysis of dECM-fibrin gels. A) Scanning electron microscopy image of the dECM-fibrin hydrogel. B-D) dECM was labeled with rhodamine isothiocyanate and gelled together with fibrin. Confocal images were taken after B) 1 day, C) 7 days, and D) 14 days, E) the dECM-fibrin hydrogel without rhodamine-labelling. The distribution of dECM is locally heterogeneous. No significant loss of dECM occurs during this time. Scale bar: $100 \mu m$.
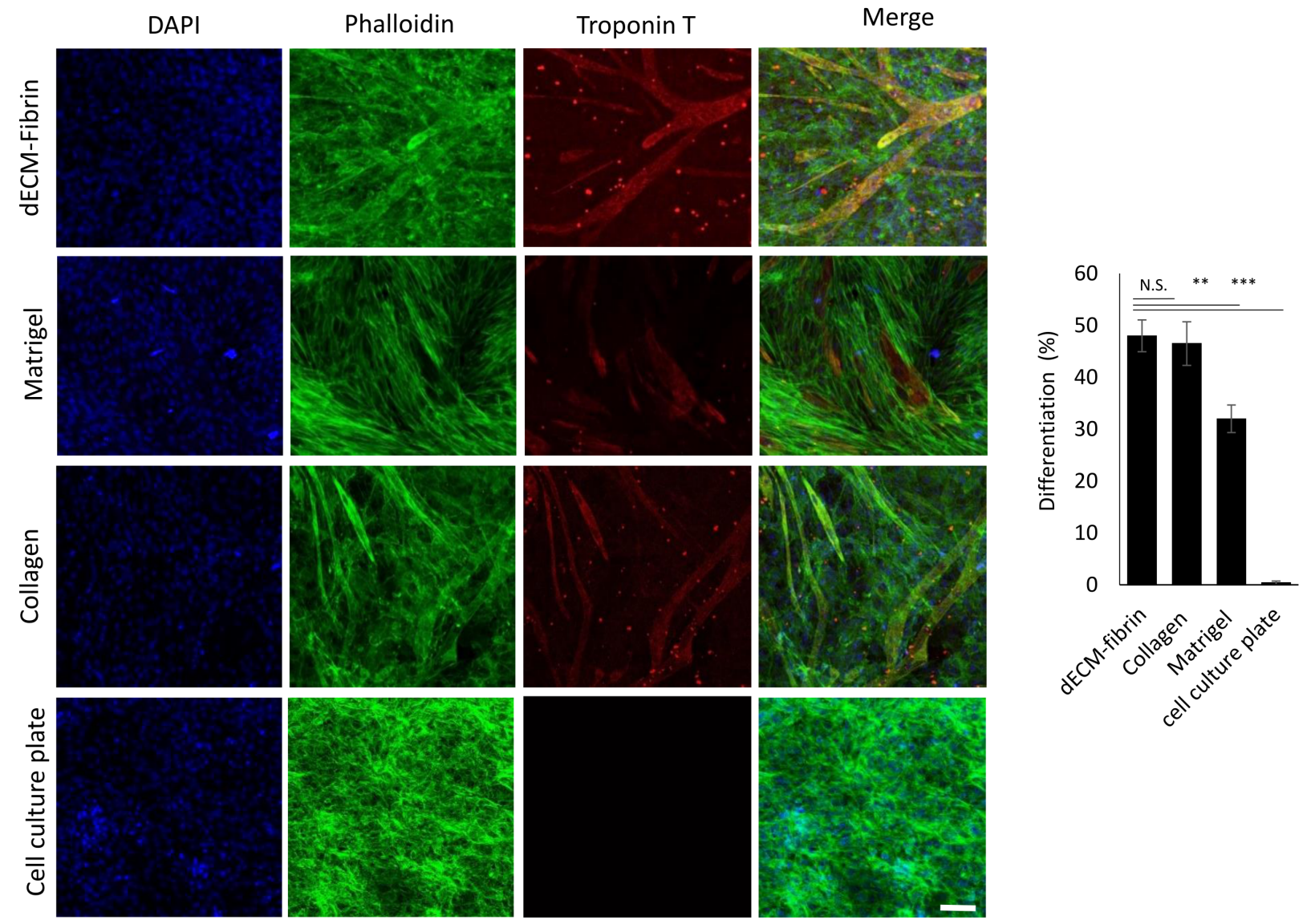

Figure 4 Cifferentiation of $\mathrm{H} 9 \mathrm{c2}$ cells in co-culture with fibroblasts on different hydrogels in the absence of retinoic acid. Differentiation A) on dECM-fibrin, B) on Matrigel, C) on collagen I, and D) on cell culture plates after 7 days of differentiation E) Percentage of differentiation of H9c2 cells on different hydrogels. Troponin $T$ staining in red shows the differentiated cells, phalloidin stains the actin filaments of both fibroblasts and H9c2 cells in green. DAPI stains the nuclei of the cells. Scale bar: $100 \mu \mathrm{m}$ ( $P=0.0024$ for dECM-fibrin vs. Matrigel, $P=0.0001$ for dECM-fibrin vs. cell culture plate, , and $P=0.645$ for $d E C M$-fibrin vs. collagen). 
bioRxiv preprint doi: https://doi.org/10.1101/2020.01.30.927319; this version posted January 31, 2020. The copyright holder for this preprint (which was not certified by peer review) is the author/funder. All rights reserved. No reuse allowed without permission.
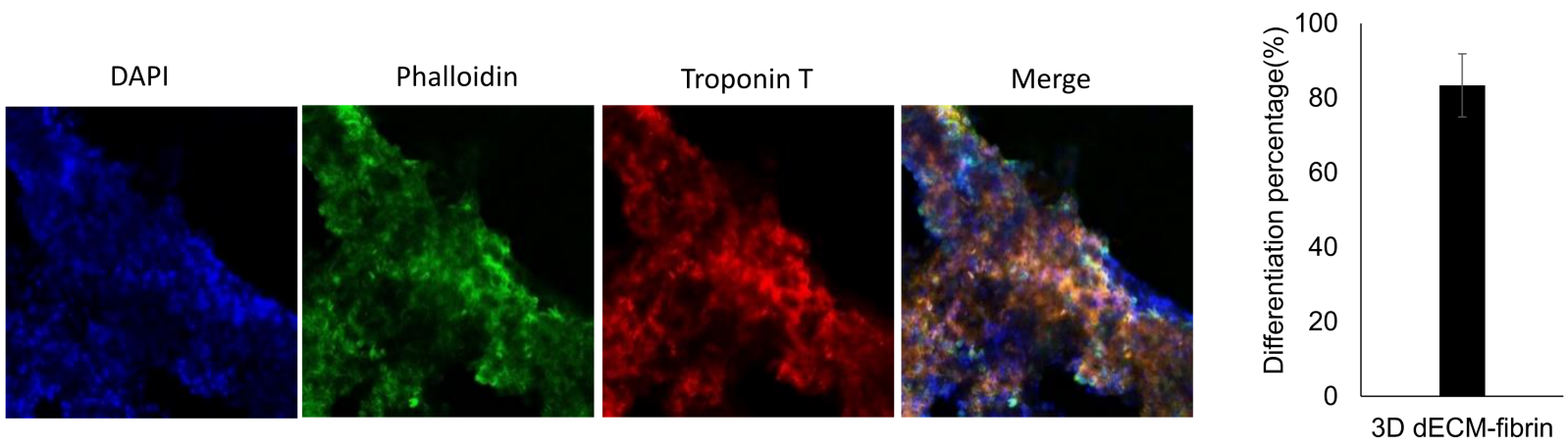

Figure 5. Co-culture of $30 \%$ of $\mathrm{H} 9 \mathrm{c2}$ cells and $70 \%$ fibroblasts in a 3D dECM-fibrin hydrogel. A) Confocal images of the differentiation of $\mathrm{H9c2}$ cells in the dECM-fibrin hydrogel after 7 days (differentiation duration). Troponin $T$ staining shows the differentiated cells in red. Phalloidin stains the actin filaments of all the cells in green and DAPI in blue stains the nuclei. Scale bar: $100 \mu m$. B) Percentage of differentiation of the H9c2 cells in the co-culture with fibroblast cells in the 3D dECM-fibrin hydrogel after 7 days (differentiation duration). 

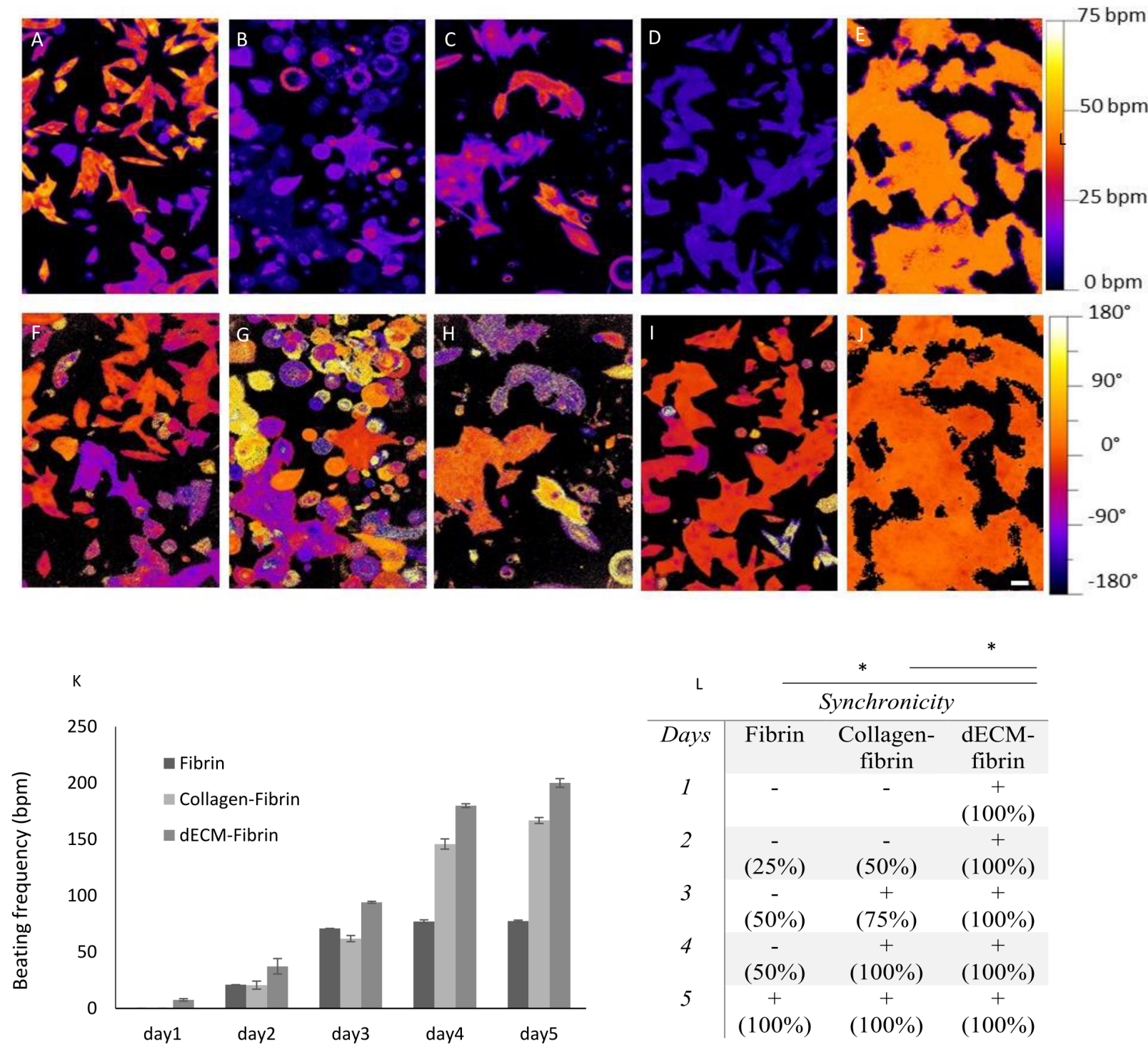

\begin{tabular}{|c|c|c|c|}
\hline & \multirow{2}{*}{\multicolumn{3}{|c|}{ Synchronicity }} \\
\hline & & & \\
\hline Days & Fibrin & $\begin{array}{l}\text { Collagen- } \\
\text { fibrin }\end{array}$ & $\begin{array}{l}\text { dECM- } \\
\text { fibrin }\end{array}$ \\
\hline 1 & - & - & $\begin{array}{c}+ \\
(100 \%)\end{array}$ \\
\hline 2 & $(25 \%)$ & $(50 \%)$ & $\begin{array}{c}+ \\
(100 \%)\end{array}$ \\
\hline 3 & $(50 \%)$ & $\begin{array}{c}+ \\
(75 \%)\end{array}$ & $\begin{array}{c}+ \\
(100 \%)\end{array}$ \\
\hline 4 & $\begin{array}{c}- \\
(50 \%)\end{array}$ & $\begin{array}{c}+ \\
(100 \%)\end{array}$ & $\begin{array}{c}+ \\
(100 \%)\end{array}$ \\
\hline 5 & $\begin{array}{c}+ \\
(100 \%)\end{array}$ & $\begin{array}{c}+ \\
(100 \%)\end{array}$ & $\begin{array}{c}+ \\
(100 \%)\end{array}$ \\
\hline
\end{tabular}

Figure 6 Calcium transients and beating characteristics of cardiomyocytes interacting with different hydrogels. A-J) Calcium imaging for neonatal cardiomyocytes seeding onto different hydrogels, and tissue culture plate (control). A-E) Frequency, F-J) Phase (positive values indicate earlier beating, negative value retardation). Hydrogels used in calcium imaging: tissue culture control $(A$ and $F)$, matrigel ( $B$ and $G)$, collagen I ( $C$ and $H)$, fibrin-collagen I ( $D$ and I) and dECM-fibrin (E and J). K) Beating rate for the first 5 days for neonatal cardiomyocytes seeded 3D in fibrin, fibrin-collagen I and dECM-fibrin hydrogels. L) Synchronization as the percentage of synchronously beating wells (four wells per condition) during 5 days in the 3D hydrogels. Scale bar: $100 \mu$ m. 
bioRxiv preprint doi: https://doi.org/10.1101/2020.01.30.927319; this version posted January 31, 2020. The copyright holder for this preprint (which was not certified by peer review) is the author/funder. All rights reserved. No reuse allowed without permission.

A

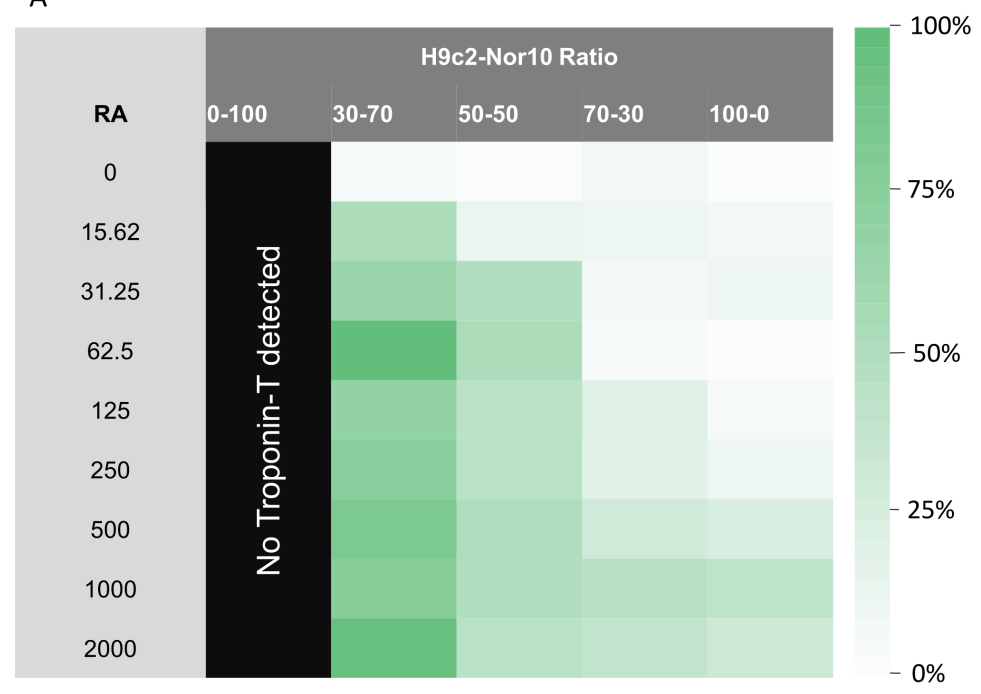

B

RA Concentration

$(n M)$

H9c2/ Fibroblast cells

\begin{tabular}{c|cc}
\hline & $30 / 70$ & $100 / 0$ \\
62.5 & $7 \pm 0.87$ & $5 \pm 0.69$ \\
2000 & $81 \pm 11.89$ & $4 \pm 1.03$ \\
& $79 \pm 10.43$ & $28 \pm 5.71$
\end{tabular}

Table 1. Differentiation of $H 9 \mathrm{c} 2$ cells in mono- and co-culture on tissue culture plates. A) The heat map of normalized differentiation percentage of $H 9 c 2$ cells for different concentrations of retinoic acid and for different ratios of H9c2 to fibroblast cells. Cultures in 2D, without hydrogel, for 7 days (differentiation duration). B) Selected numerical values for the differentiation percentages for the 30/70 ratio and 100/0 ratio. 